\title{
Indução de ivermectina na hormese sobre Eisenia foetida durante a vermicompostagem de esterco bovino
}

\author{
Sandro M. Tuerlinckx ${ }^{1}$, Tânia B. G. A. Morselli² \& Ana C. K. Huber ${ }^{3}$ \\ ${ }^{1}$ Centro de Ciências da Saúde/Universidade da Região da Campanha. Bagé, RS. E-mail: sandromtuer@gmail.com (Autor correspondente) \\ ${ }^{2}$ PPG Sistemas de Produção Agrícola Familiar/Faculdade de Agronomia Eliseu Maciel/Universidade Federal de Pelotas. Pelotas, RS. E-mail: tamor@uol.com.br \\ ${ }^{3}$ Centro de Ciências Exatas e Ambientais/Universidade da Região da Campanha. Bagé, RS. E-mail: anackalil@hotmail.com
}

\section{Palavras-chave:}

efeito hormético

fármacos veterinários

minhocas

toxicidade

\begin{abstract}
R E S U M O
Os medicamentos veterinários são, em sua maioria, excretados nas fezes de animais tratados demonstrando que os fármacos da classe das avermectinas podem ter efeitos nocivos sobre os organismos não alvo, caso se utilizem das fezes. O objetivo deste estudo foi determinar os efeitos subletais da ivermectina sobre as minhocas Eisenia foetida expostas às concentrações de ivermectina a $0,1,5,10,50$ e $100 \mathrm{mg} \mathrm{kg}^{-1}$; amostras foram tomadas nos dias 7,14 e 28 de exposição para determinação da sobrevivência, crescimento, produção de casulos e atividades enzimáticas, além de estudadas as alterações químicas e a atividade microbiana do vermicomposto não se observando, no entanto, efeitos negativos da ivermectina quanto à mortalidade de minhocas; da mesma forma, as características químicas e a atividade microbiana do vermicomposto não foram afetadas porém os resultados demonstraram que a ivermectina induz, quando em baixas concentrações, a um aumento na atividade da fosfatase alcalina e no crescimento de E. foetida mas altas concentrações inibiram esses parâmetros, o que foi refletido numa curva em forma de U invertido, uma representação gráfica do efeito hormético.
\end{abstract}

Key words:

earthworms

hormetic effect

toxicity

veterinary pharmaceuticals

\section{Induction of ivermectin in the hormesis on Eisenia foetida during the vermicomposting of cattle manure}

\begin{abstract}
A B S T R A C T
Most of the veterinary pharmaceuticals are excreted in the faeces of treated animals and it has been demonstrated that members of the avermectin group may have deleterious effects on non-target organisms utilising the faeces. The aim of this study is to determine the sublethal effects of ivermectin on the Eisenia foetida earthworms. Earthworms were exposed to ivermectin at $0,1,5,10,50$ and $100 \mathrm{mg} \mathrm{kg}^{-1}$ concentrations; samples were taken at 7, 14, and 28 days exposure for determination of survival, growth rate, cocoon production and enzyme activities. Furthermore, the chemical changes and microbial activity of the vermicompost were also studied. There was no negative effects of ivermectin on mortality of earthworms. Likewise the chemical characteristics and microbial activity of vermicompost were not affected. However, the results showed that ivermectin at low concentrations induces an increase in the activity of alkaline phosphatase and growth of E. foetida, but high concentrations inhibited these endpoints, and this was reflected in an inverted U-shaped curve, a graphic representation of hormetic effect.
\end{abstract}




\section{INTRODUÇÃO}

A vermicompostagem é de fundamental importância na atualidade visto que pode ser utilizada como forma de biorremediação para promover a biodegradação de contaminantes orgânicos e a reciclagem dos resíduos de fontes agroindustriais (Silva et al., 2002; Yadav \& Garg, 2011).

É um processo bioquímico que tem a participação combinada de micro-organismos e minhocas que atuam na fragmentação e na degradação da matéria orgânica (Prabha et al., 2007; Ueda et al., 2008; Aira \& Domínguez, 2009) possibilitando o reaproveitamento do esterco bovino, um resíduo sólido produzido em elevada quantidade durante o sistema de pecuária intensiva (Garg et al., 2006). Entretanto, a presença de vários compostos e metabólitos oriundos de fármacos veterinários $\mathrm{e}$ que são eliminados nas fezes de animais tratados, incide num risco ecológico no uso dos estercos (Floate et al., 2005) podendo, inclusive, comprometer o processo de vermicompostagem.

A ivermectina, uma lactona macrocíclica pertencente à classe das avermectinas, é comumente e globalmente aplicada para eliminar ectoparasitas e nematoides gratintestinais de uma variedade de espécies animais; sofre pouca metabolização e é parcialmente excretada de forma inalterada pelas fezes de animais tratados (Lumaret et al., 2012).

Dependendo da formulação comercial de ivermectina utilizada, as concentrações ambientais do fármaco podem atingir até $20 \mathrm{mg} \mathrm{kg}^{-1}$ peso seco (Floate et al., 2005), persistindo por dias a meses tanto em estercos como no solo (Iglesias et al., 2006; Krogh et al., 2009).

Tem-se demonstrado que a presença de resíduos ambientais de fármacos veterinários, principalmente da classe das avermectinas, afeta organismos não-alvo que habitam os estercos e o solo, levando as agências internacionais de regulação ambiental a emitir alertas sobre o risco de substâncias potencialmente tóxicas excretadas por animais de produção (Lumaret et al., 2012; Blanckenhorn et al., 2013).

Embora as pesquisas conduzidas por Kaneda et al. (2006) apontem que as concentrações ambientalmente relevantes de ivermectina não afetam as minhocas, outros estudos demonstraram que este endectocida prejudica os aspectos reprodutivos, como a produção e a eclodibilidade dos casulos (Gunn \& Sadd 1994; Rombke et al., 2009) enquanto que a mortalidade só é evidenciada em concentrações elevadas, entre $56 \mathrm{mg} \mathrm{kg}^{-1}$ (Wang et al., 2012) e $314 \mathrm{mg} \mathrm{kg}^{-1}$ (Halley et al., 1989).

Recentemente, tem-se observado uma reemergência de estudos científicos em relação a um fenômeno conhecido como efeito hormético (Calabrese, 2005).

A hormese é um conceito toxicológico que se refere a uma dose-resposta bifásica (não monotônica) a um agente ambiental e que é caracterizada pela estimulação em baixas concentrações e uma inibição em concentrações elevadas (Calabrese \& Blain, 2011). Entretanto, a detecção correta e a estimação deste efeito dependem da aplicação de modelos estatísticos adequados (Deng et al., 2001; Zhu et al., 2013). Desta forma, a relação entre a área sob as curvas hormética e do ponto de equivalência zero $\left(\mathrm{AUC}_{\mathrm{H}} / \mathrm{AUC}_{\mathrm{ZEP}}\right)$ pode ser usada como estimativa da magnitude do efeito hormético e também identificar os parâmetros que desempenham papel relevante neste processo (Deng et al., 2001).
Em minhocas Eisenia foetida tem-se demonstrado que elementos químicos, como o cádmio e compostos orgânicos da classe dos organofosforados, induzem efeito hormético sobre enzimas anticolinérgicas e antioxidantes (Hackenberger et al., 2008; Zhang et al., 2009); no entanto, este efeito ainda não foi estudado em organismos expostos à IVM.

Objetivou-se, no presente estudo, verificar se concentrações subletais de ivermectina induzem hormese sobre o desenvolvimento, produção de casulos e atividades enzimáticas de Eisenia foetida durante o processo de vermicompostagem de esterco bovino e determinar se este endectocida altera a atividade microbiana e os atributos químicos do vermicomposto.

\section{Material e Métodos}

As condições metodológicas da pesquisa seguiram as recomendações descritas por Gao et al. (2007) utilizando-se, como substrato teste, esterco bovino livre de urina e oriundo de animais que não tiveram nenhum tipo de tratamento químico por pelo menos sessenta dias. As características químicas do substrato utilizado foram: umidade $70 \%$; $\mathrm{pH} 8,4$; carbono orgânico 465,3 $\mathrm{g} \mathrm{kg}^{-1}$; nitrogênio total $11,2 \mathrm{~g} \mathrm{~kg}^{-1}$; fósforo total 3,5 $\mathrm{g} \mathrm{kg}^{-1}$; potássio total $6,6 \mathrm{~g} \mathrm{~kg}^{-1}$; cálcio total $13,5 \mathrm{~g} \mathrm{~kg}^{-1}$; magnésio total $4,4 \mathrm{~g} \mathrm{~kg}^{-1}$ e a relação C/N 41:1.

A ivermectina (Lote 071M1916), adquirida da empresa Sigma-Aldrich Brasil Ltda, São Paulo - SP, Brasil, com grau de pureza superior a $98 \%$, foi dissolvida inicialmente em acetona e em seguida misturada com o substrato para atingir as concentrações de 0, 1, 5, 10, 50 e $100 \mathrm{mg} \mathrm{kg}^{-1}$ de peso seco; posteriormente foi adicionada, à mistura, água destilada para atingir o equivalente em peso úmido de $75 \%$.

Foram preparadas quatro repetições para cada concentração, com 10 minhocas pesadas individualmente $(330 \pm 2 \mathrm{mg}$, servindo como 10 réplicas) e adicionadas em cada recipiente os quais foram mantidos a $21 \pm 2{ }^{\circ} \mathrm{C}$ em fotoperíodo de $12 \mathrm{~h}$ luz 12 $\mathrm{h}^{-1}$ escuro, com intensidade luminosa de 400-800 lux e umidade de 75\% em câmara climatizada modelo 347 CDG - FANEM/SP. As minhocas utilizadas neste ensaio foram adultas com clitelo desenvolvido e peso entre 300-400 mg.

A mortalidade da $E$. foetida foi determinada pela contagem do número de minhocas não sobreviventes aos 7, 14 e 28 dias do experimento. As minhocas eram consideradas mortas quando não possuíam movimento e não respondiam a nenhum estímulo tátil definido até a porção final anterior.

Os pesos das minhocas nos diferentes grupos de concentração testadas foram relatados a partir do início do período de exposição e usados para calcular a taxa de desenvolvimento.

$\mathrm{O}$ efeito da ivermectina sobre a capacidade reprodutiva de Eisenia foetida foi avaliado mediante a produção de casulos; após o período de exposição de 28 dias, as minhocas foram retiradas do substrato e contado manualmente o número de casulos produzidos em cada repetição.

Vinte e quatro horas antes da realização das análises bioquímicas as minhocas foram retiradas dos substratos teste e colocadas em placas de Petri sobre papel filtro Whatman $n^{\circ} 1$ umedecido na temperatura de $21 \pm 2{ }^{\circ} \mathrm{C}$ com luz $12: 12 \mathrm{~h}$ : ciclo claro-escuro, para esvaziamento do conteúdo do trato digestório e em seguida armazenadas em nitrogênio líquido no Laboratório 
de Bioquímica e Toxicologia Animal - CCR/URCAMP-Bagé, RS; posteriormente, as minhocas foram homogeneizadas em tampão Tris- $\mathrm{HCl}\left(0,1 \mathrm{Mol} \mathrm{L}^{-1}, \mathrm{pH} 7,6\right)$ em quatro partes (4:1 peso:volume), contendo fluoreto de fenilmetilsulfonil (PMSF; 0,1 $\mathrm{mmol} \mathrm{L}^{-1}$ ) como inibidor de proteases usando-se um homogeneizador de tecidos Heidolph a $6.000 \mathrm{rpm}$; em seguida, o homogeneizado foi centrifugado a $3.000 \mathrm{~g}$ por $30 \mathrm{~min}$ em uma centrífuga Coleman modelo 90-1, resultando em um sobrenadante $(S 1)$ que foi utilizado para a medição das atividades enzimáticas; enfim, todos os preparos teciduais foram realizados a $4^{\circ} \mathrm{C}$.

O conteúdo proteico total dos sobrenadantes foi determinado pela reação de biureto utilizando-se um kit comercial (Labtest, Lagoa Santa, MG, Brasil) realizado em triplicatas a $545 \mathrm{~nm}$.

As avaliações das atividades das enzimas $\alpha$-amilase, fosfatase alcalina e aspartato aminotransferase foram realizadas utilizando-se kit bioquímico da Biotécnica Ltda. (Varginha, MG). A atividade específica de cada enzima foi expressa como $\mu \mathrm{mol}$ do produto formado $\mathrm{mg}^{-1}$ proteína $\mathrm{min}^{-1}$.

A atividade microbiana foi avaliada nos grupos experimentais nos dias $0,7,14,21$ e 28 pela taxa de evolução do $\mathrm{CO}_{2}$ da amostra durante $24 \mathrm{~h}$ de incubação. $\mathrm{O} \mathrm{CO}_{2}$ foi capturado em uma solução de $\mathrm{NaOH}$ 0,05 mol L-1 e subsequentemente mensurado pela titulação da fenolftaleína com $\mathrm{HCl}$, após adição de excesso de $\mathrm{BaCl}_{2}$. A atividade microbiana foi expressa como $\mu \mathrm{g} \mathrm{CO}_{2} \mathrm{~g}^{-1}$ vermicomposto $\mathrm{h}^{-1}$ (Atiyeh et al., 2000).

O substrato inicial (esterco bovino) e os vermicompostos obtidos no experimento foram analisados para suas características químicas no Laboratório de Química do Departamento de Solos da FAEM/UFPel, no qual foram determinados: $\mathrm{pH}$, umidade, nitrogênio, fósforo, potássio, cálcio, magnésio, carbono e a relação $\mathrm{C} / \mathrm{N}$ cujos métodos utilizados foram descritos por Tedesco et al. (1995).

Os testes paramétricos foram precedidos por ShapiroWilk para distribuição normal e pelo teste de Levene para a homogeneidade das variâncias. Diferenças significativas foram testadas pela análise de variância (ANOVA) e as comparações post hoc foram realizadas pelo teste de Duncan para as médias entre os grupos expostos e o grupo controle. As diferenças entre os grupos foram consideradas significantes a $\mathrm{P}<0,05$, utilizando-se o programa IBM SPSS 20.
A determinação da atividade enzimática relativa foi realizada pela transformação dos dados da atividade enzimática específica como percentual (\%) em relação ao grupo controle (dose $0 \mathrm{mg}$ $\mathrm{kg}^{-1}$ ) através da fórmula: (valor obtido - valor do controle/valor do controle) ${ }^{\star} 100$, conforme Zhang et al. (2009).

Adicionalmente, foi aplicada a função cúbica sobre os dados obtidos para descrever uma relação dose-resposta à incorporação de efeito hormético. A extensão da hormese foi estimada de acordo com Deng et al. (2001) como demonstrado na Eq. 1, pela relação de $\mathrm{AUC}_{\mathrm{H}} / \mathrm{AUC}_{\mathrm{ZEP}}$ utilizando-se o método integral direto, através do programa SIGMA PLOT 10.0.

$$
P=\frac{\mathrm{AUC}_{\mathrm{H}}}{A \mathrm{AC}_{\mathrm{ZEP}}} \cdot 100 \%=\frac{\int_{\mathrm{ZEP}_{1}}^{\mathrm{ZEP}_{2}} \mathrm{f}(\mathrm{x}) \mathrm{dx}-\mathrm{Y}_{0}\left(\mathrm{ZEP}_{2}-\mathrm{ZEP}_{1}\right)}{\int_{\mathrm{ZEP}_{1}}^{\mathrm{ZEP}_{2}} \mathrm{f}(\mathrm{x}) \mathrm{dx}} \cdot 100
$$

\section{Resultados e Discussão}

Não foi observada mortalidade de minhocas em nenhum dos tratamentos, ao longo dos 28 dias de exposição à IVM em esterco bovino utilizado como substrato teste; desta forma, a $\mathrm{CL}_{50}$ foi determinada como $>100 \mathrm{mg} \mathrm{kg}^{-1}$ substrato (peso seco), confirmando o carácter de subletalidade das concentrações testadas.

Resultados contraditórios são encontrados em testes de toxicidade da IVM sobre E. foetida. Em sua pesquisa, Halley et al. (1989) demonstraram que a $\mathrm{CL}_{50}$ de IVM é de $314 \mathrm{mg}$ $\mathrm{kg}^{-1}$ solo; contudo, Gunn \& Sadd (1994) observaram não haver sobrevivência em solos quando a concentração de IVM alcança $16 \mathrm{mg} \mathrm{kg}^{-1}$ da mesma forma, Sun et al. (2005) verificaram mortalidade a $24.1 \mathrm{mg} \mathrm{kg}^{-1} \mathrm{e}$ Wang et al. (2012) a $68.11 \mathrm{mg} \mathrm{kg}^{-1}$ solo (peso seco) para a mesma substância.

Os resultados referentes à taxa de desenvolvimento, produção de casulos e atividades enzimáticas específicas da AST, FALC e AMIL de minhocas E. foetida expostas a diferentes concentrações de IVM durante o período experimental, podem ser observados como valores da média, desvio padrão e indicação de diferenças significativas pelo teste de Duncan a 0,05\% de significância (Tabela1).

Tabela 1. Parâmetros relacionados à Eisenia foetida e a atividade microbiana do vermicomposto após 7, 14 e 28 dias de exposição às várias concentrações de ivermectina (IVM)

\begin{tabular}{|c|c|c|c|c|c|c|c|}
\hline \multirow{2}{*}{ Parâmetro } & \multirow{2}{*}{$\begin{array}{l}\text { Exposição } \\
\text { (dias) }\end{array}$} & \multicolumn{6}{|c|}{ Concentração IVM (mg kg-1 substrato) } \\
\hline & & 0 & 1 & 5 & 10 & 50 & 100 \\
\hline \multirow{3}{*}{$\begin{array}{l}\text { Desenvolvimento } \\
(\%)\end{array}$} & 7 & $25 \pm 8,8 \mathrm{a}$ & $25 \pm 9,6 a$ & $28 \pm 2,2 \mathrm{a}$ & $39 \pm 4,6 b$ & $10 \pm 3,1 c$ & $-4 \pm 7,1 d$ \\
\hline & 14 & $44 \pm 5,1 a b$ & $47 \pm 8,4 a$ & $52 \pm 0,9 a$ & $56 \pm 8,1 \mathrm{a}$ & $33 \pm 0,6 b$ & $-2 \pm 8,9 c$ \\
\hline & 28 & $50 \pm 8,8 b$ & $72 \pm 9,3 a$ & $68 \pm 9,9 a b$ & $61 \pm 13,7 \mathrm{ab}$ & $66 \pm 7,9 a b$ & $50 \pm 2,8 b$ \\
\hline Casulos (produção média minhoca ${ }^{-1}$ ) & 28 & $8 \pm 0,6 a b$ & $8,4 \pm 1,5 \mathrm{a}$ & $9,2 \pm 0,2 a$ & $8,4 \pm 0,6 \mathrm{a}$ & $6,6 \pm 0,4 b$ & $2,5 \pm 1 c$ \\
\hline \multirow{3}{*}{$\begin{array}{l}\text { Atividade AST } \\
\left(\mu \text { mol NADH mg-1 proteína min }{ }^{-1}\right)\end{array}$} & 7 & $8,7 \pm 0,4 a$ & $8,6 \pm 1,2 \mathrm{a}$ & $8,8 \pm 0,5 a$ & $8,5 \pm 0,9 a$ & $8,5 \pm 0,2 a$ & $8,5 \pm 0,8 \mathrm{a}$ \\
\hline & 14 & $8,6 \pm 0,5 a$ & $8,7 \pm 1,2 \mathrm{a}$ & $8,7 \pm 0,5 a$ & $8,6 \pm 0,9 a$ & $8,5 \pm 0,2 a$ & $8,4 \pm 0,9 a$ \\
\hline & 28 & $9,8 \pm 0,6 \mathrm{a}$ & $9,7 \pm 1,7 \mathrm{a}$ & $8,7 \pm 0,4 a$ & $8,6 \pm 1,2 a$ & $8,5 \pm 0,9 \mathrm{ab}$ & $6,9 \pm 1,4 b$ \\
\hline \multirow{3}{*}{$\begin{array}{l}\text { Atividade FALC } \\
\left(\mu \mathrm{mol} \text { PNF } \mathrm{mg}^{-1} \text { proteína } \text { min }^{-1}\right)\end{array}$} & 7 & $10,4 \pm 0,9 a$ & $11,3 \pm 1,1 \mathrm{a}$ & $11,7 \pm 2,4 a$ & $11,2 \pm 0,8 \mathrm{a}$ & $9,9 \pm 1,7 a$ & $9,7 \pm 0,2 a$ \\
\hline & 14 & $7,7 \pm 1,1 \mathrm{ab}$ & $8,5 \pm 0,8 a b$ & $9 \pm 1,3 \mathrm{a}$ & $9 \pm 1,4 \mathrm{a}$ & $7,8 \pm 1,1 \mathrm{ab}$ & $6,3 \pm 1,4 b$ \\
\hline & 28 & $7,7 \pm 1,3 \mathrm{ab}$ & $8,4 \pm 0,7 \mathrm{a}$ & $8,9 \pm 1,1 \mathrm{a}$ & $8,2 \pm 2,1 \mathrm{ab}$ & $7,7 \pm 1,2 a b$ & $6,1 \pm 1,3 b$ \\
\hline \multirow{3}{*}{$\begin{array}{l}\text { Atividade AMIL } \\
\left(\mu \mathrm{mol} \text { CNP } \mathrm{mg}^{-1} \text { proteína } \mathrm{min}^{-1}\right)\end{array}$} & 7 & $9,9 \pm 1,2 \mathrm{a}$ & $9,1 \pm 0,9 a$ & $8,8 \pm 1,7 \mathrm{a}$ & $8,9 \pm 2,1 \mathrm{a}$ & $9,5 \pm 0,6 a$ & $9,1 \pm 0,9 a$ \\
\hline & 14 & $8,9 \pm 0,7 a$ & $9,2 \pm 0,7 a$ & $10,1 \pm 0,8 a$ & $10,9 \pm 1 \mathrm{a}$ & $10,4 \pm 1 \mathrm{a}$ & $10,2 \pm 1 \mathrm{a}$ \\
\hline & 28 & $11,3 \pm 2,9 a$ & $11,4 \pm 1,9 a$ & $11,1 \pm 1,4 \mathrm{a}$ & $10,3 \pm 0,8 \mathrm{a}$ & $9,5 \pm 2,8 \mathrm{a}$ & $9,4 \pm 0,9 a$ \\
\hline \multirow{3}{*}{$\begin{array}{l}\text { Atividade microbiana } \\
\left(\mu \mathrm{g} \mathrm{CO}_{2} \mathrm{~g}^{-1} \text { substrato } \mathrm{h}^{-1}\right)\end{array}$} & 7 & $0,25 \pm 0,3 a$ & $0,25 \pm 0,4 a$ & $0,24 \pm 0,3 a$ & $0,26 \pm 0,5 \mathrm{a}$ & $0,25 \pm 0,4 a$ & $0,24 \pm 0,3 a$ \\
\hline & 14 & $0,23 \pm 0,4 \mathrm{a}$ & $0,22 \pm 0,3 \mathrm{a}$ & $0,23 \pm 0,3 a$ & $0,24 \pm 0,5 \mathrm{a}$ & $0,23 \pm 0,6 a$ & $0,22 \pm 0,3 \mathrm{a}$ \\
\hline & 28 & $0,22 \pm 0,5 \mathrm{a}$ & $0,23 \pm 0,3 a$ & $0,23 \pm 0,5 a$ & $0,24 \pm 0,3 \mathrm{a}$ & $0,21 \pm 0,4 a$ & $0,22 \pm 0,5 \mathrm{a}$ \\
\hline
\end{tabular}

Médias seguidas de letras iguais na linha não diferem entre si pelo teste de Duncan $(p<0,05)$; AST - Aspartato aminotransferase; FALC - Fosfatase alcalina; AMIL - Amilase 
Dependendo da formulação comercial de ivermectina, após sua metabolização e excreção as concentrações ambientalmente relevantes do fármaco no esterco podem alcançar até $20 \mathrm{mg} \mathrm{kg}^{-1}$ peso seco (Floate et al., 2005).

De acordo com as condições metodológicas utilizadas neste estudo, a IVM em baixas concentrações não afeta negativamente a taxa de desenvolvimento nem a atividade microbiana (Tabela 1 ), corroborando com as pesquisas conduzidas por Kaneda et al. (2006) e Iglesias et al. (2011), porém Gunn \& Sadd (1994) encontraram prejuízo no crescimento de minhocas expostas durante 14 dias a 4,7 $\mathrm{mg} \mathrm{kg}^{-1}$ de IVM; do mesmo modo, Halley et al. (1989) demonstraram diminuição significativa na biomassa de E. foetida aos 28 dias de exposição a $12 \mathrm{mg} \mathrm{kg}^{-1}$.

Por outro lado e como demonstrado na Tabela 1 , nas duas primeiras semanas do experimento foi verificada diminuição na taxa de desenvolvimento caracterizada pela redução no peso das minhocas expostas às duas concentrações mais elevadas de IVM. Este evento pode estar relacionado ao mecanismo de ação do fármaco em invertebrados que atuam mediante a ligação irreversível e ativação de canais iônicos dependentes de ligantes, particularmente canais cloretos dependentes de glutamato e receptores do ácido $\gamma$-aminobutírico (GABA), resultando em paralisia corporal e disfunção dos músculos da faringe (Lumaret et al., 2012).

Uma condição observada neste experimento foi que a IVM induziu efeito hormético sobre a taxa de desenvolvimento de E. foetida.

A Figura 1 demonstra a estimação da hormese para o parâmetro taxa de desenvolvimento em minhocas $E$. foetida expostas a diferentes concentrações de IVM, ao longo de 28 dias do experimento.

Sete dias após o início do experimento os resultados observados para o coeficiente $\beta_{1}$ demonstram significância a nível $\alpha=0,05(\mathrm{t}=3,22, \mathrm{p}<0,01)$ indicando que a IVM induz efeito hormético sobre a taxa de desenvolvimento de E. foetida durante este período de exposição. A função cúbica pode ser expressa como: $y=23,2251+2,0788 x-0,0699 x^{2}+0,0005 x^{3}\left(R^{2}\right.$ $=0,86, \mathrm{~F}=27,68, \mathrm{p}<0,01)$ e foi utilizada visando à obtenção do $\mathrm{ZEP}\left(\mathrm{ZEP}_{1}=-8,56 ; \mathrm{ZEP}_{2}=82,85\right)$.
Utilizando o método integral direto descrito por Deng et al. (2001) foram calculadas a área hormética $\mathrm{AUC}_{\mathrm{H}}$ como 653,1, a $\mathrm{AUC}_{\mathrm{ZEP}}$ como 1676,6 e a razão entre as duas áreas 38,9\%. O efeito hormético máximo obtido aos 7 dias de exposição à IVM foi $Y \max =32,9 \%$.

A hormese se prolongou até o $14^{\circ}$ dia de exposição da $E$. foetida em diferentes concentrações de IVM. O coeficiente $\beta_{1}$, indicativo de efeito hormético, foi significativo a nível $\alpha=0,05$ $(\mathrm{t}=2,79, \mathrm{p}<0,05)$ e a função cúbica pode ser expressa como: $\mathrm{y}$ $=44,6787-1,6305 x-0,0537 x^{2}+0,0003 x^{3}\left(R^{2}=0,93, F=63,68\right.$, $\mathrm{p}<0,01)$ e só então utilizada para obtenção do ZEP $\left(\mathrm{ZEP}_{1}=\right.$ -16,9; $\left.\mathrm{ZEP}_{2}=102,1\right)$. Desta forma, a área hormética $\mathrm{AUC}_{\mathrm{H}}$ calculada como 1957,8 , a AUC $_{\text {ZEP }}$ como 3242,5 e a razão entre as duas áreas $60,4 \%$. O efeito hormético máximo obtido aos 14 dias de exposição a IVM foi $Y \max =56,5 \%$, comparativamente maior que no sétimo dia, como pode ser verificado na Figura 1.

A IVM não induziu, entretanto, a hormese aos 28 dias sobre a taxa de desenvolvimento de E. foetida; como verificado pelo coeficiente $\beta_{1}$ não houve significância a nível $\alpha=0,05(t=0,44$, $\mathrm{p}=0,67)$, sendo a função cúbica expressa como: $\mathrm{y}=61,1361+$ $0,4948 \mathrm{x}-0,0100 \mathrm{x}^{2}+0,00005 \mathrm{x}^{3}\left(\mathrm{R}^{2}=0,21, \mathrm{~F}=1,27, \mathrm{p}=0,3218\right)$. Essas observações estão de acordo com os dados disponíveis na literatura indicando que o efeito hormético apresenta carácter transitório e que não é apenas uma simples relação dose-resposta, mas também uma relação dose-tempo-resposta (Cedergreen, 2008).

Incrementos na taxa de desenvolvimento de minhocas alimentadas com esterco de bovinos que foram tratados com ivermectina, também foram relatados por Svendesen et al. (2005).

Não foi constatado efeito hormético $(\mathrm{t}=0,84, \mathrm{p}=0,41)$ para a produção de casulos porém ocorreu uma redução significativa (ANOVA, p < 0,05) evidenciada somente na concentração mais elevada (100 mg kg-1). Diferentemente, Gunn \& Sadd (1994) verificaram que a concentração de apenas $4 \mathrm{mg} \mathrm{kg}^{-1}$ de IVM reduziu a produção de casulos em 56\% durante 21 dias em solo artificial.

As distintas concentrações de ivermectina não produziram alterações significativas sobre a atividade específica da enzima

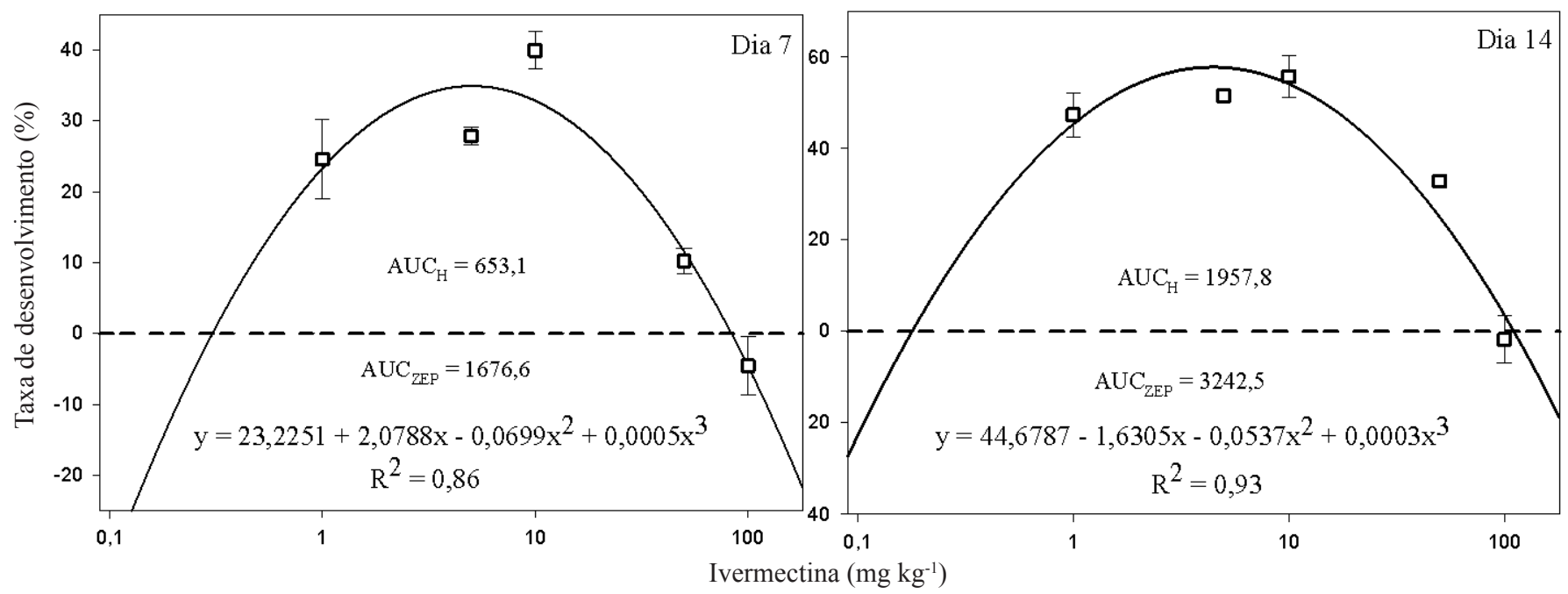

Figura 1. Efeito hormético sobre a taxa de desenvolvimento em Eisenia foetida aos 7 e 14 dias de exposição a diferentes concentrações de ivermectina. Os dados são demonstrados como média \pm desvio padrão. $\mathrm{AUC}_{\mathrm{H}}$ representa a área sob a curva hormética e $\mathrm{AUC}_{\mathrm{ZEP}}$ indica a área sob a curva do ponto de equivalência zero 
AST até os 14 dias de exposição. Por outro lado, observou-se diminuição significativa (ANOVA, $\mathrm{p}<0,05$ ) desta enzima ao final do período experimental na concentração de $100 \mathrm{mg} \mathrm{kg}^{-1}$ mas este achado não foi suficiente para alterar significativamente a relação $\mathrm{C} / \mathrm{N}$ nem a concentração de nitrogênio total do vermicomposto, como pode ser observado na Tabela 2 .

As enzimas digestórias encontradas em minhocas são responsáveis pela decomposição e humificação da matéria orgânica (Prabha et al., 2007). Neste sentido, Ueda et al. (2008) caracterizaram, em minhocas $E$. foetida, dois tipos de $\alpha$-amilases com propriedades termoestáveis sugerindo que ambas as enzimas possuem funções complementares e com alta atividade hidrolítica sobre o amido. Os resultados encontrados neste trabalho revelam que a enzima $\alpha$-amilase de E. foetida não foi significativamente afetada durante os diferentes períodos de exposição à IVM.

Ao se analisar as atividades relativas da enzima FALC, constatou-se a presença de efeito hormético produzido pela IVM nos $14^{\circ}$ e $28^{\circ}$ dias de exposição sobre a E. foetida (Figura 2).

Aos 14 dias após o início do experimento os resultados observados para o coeficiente $\beta_{1}$ demonstram significância a nível $\alpha=0,05(\mathrm{t}=3,85, \mathrm{p}<0,01)$, indicando que a IVM induz hormese sobre a enzima FALC de E. foetida a partir deste período de exposição; a função cúbica pode ser expressa como: $\mathrm{y}=4,7748$ $+2,0075 \mathrm{x}-0,0608 \mathrm{x}^{2}+0,0004 \mathrm{x}^{3}\left(\mathrm{R}^{2}=0,83, \mathrm{~F}=31,61, \mathrm{p}<0,01\right) \mathrm{e}$ $\mathrm{ZEP}=50,84$. A área hormética $\mathrm{AUC}_{\mathrm{H}}$ obtida foi 345,4 e a $\mathrm{AUC}_{\text {ZEP }}$ foi 587,9, a razão $\mathrm{AUC}_{\mathrm{H}} / \mathrm{AUC}_{\mathrm{ZEP}}$ estimada em 58,7\% e o efeito hormético máximo encontrado foi $Y \max =17,1 \%$.
A enzima FALC também manifestou hormese no $28^{\circ}$ dia de exposição da E. foetida a diferentes concentrações de IVM, em que o coeficiente $\beta_{1}$ foi significativo a nível $\alpha=0,05$ ( $\mathrm{t}=$ $1,91, \mathrm{p}<0,05)$ e a função cúbica pode ser expressa como: $\mathrm{y}=$ $6,1825+1,1939 x-0,0361 x^{2}+0,0002 x^{3}\left(R^{2}=0,75, F=20,29\right.$, $\mathrm{p}<0,01)$ e $\mathrm{ZEP}=50,82$. A área hormética $\mathrm{AUC}_{\mathrm{H}}$ obtida foi 113,1 e a $\mathrm{AUC}_{\mathrm{ZEP}}$ foi 427,2 , a razão $\mathrm{AUC}_{\mathrm{H}} / \mathrm{AUC}_{\mathrm{ZEP}}$ estimada em $26,5 \%$ e o efeito hormético máximo encontrado foi Ymax $=15,9 \%$, comparativamente menor que no $14^{\circ} \mathrm{dia}$, como pode ser verificado na Figura 2.

Referidos resultados estão de acordo com a afirmação de que a resposta máxima da hormese normalmente é modesta e que diferentes parâmetros podem ser afetados em diferentes concentrações (Calabrese \& Blain, 2011).

Zhang et al. (2009) determinaram a magnitude do efeito hormético pela razão $\mathrm{AUC}_{\mathrm{H}} / \mathrm{AUC}_{\mathrm{ZEP}}$ da enzima superóxido dismutase em $52,8 \%$ e para a catalase $36,8 \%$ em minhocas $E$. foetida expostas ao cádmio.

O efeito hormético em minhocas do gênero Eisenia também foi documentado para a enzima acetilcolinesterase durante a exposição ao inseticida organofosforado temefós (Hackenberger et al., 2008).

O fenômeno da hormese está relacionado, provavelmente, com a ativação de vias metabólicas adaptativas, responsáveis pela homeostase celular e fisiológica (Calabrese \& Blain, 2011). Desta forma, o efeito hormético detectado para a enzima fosfatase alcalina pode explicar, em parte, o efeito observado para o parâmetro taxa de desenvolvimento visto que enzimas

Tabela 2. Atributos químicos do vermicomposto após 28 dias de exposição a várias concentrações de ivermectina (IVM)

\begin{tabular}{lcccccc}
\hline \multirow{2}{*}{ Parâmetro } & \multicolumn{5}{c}{ Concentração IVM (mg kg $\mathbf{~}^{-1}$ substrato) } \\
\cline { 2 - 7 } pH $\left(\mathrm{H}_{2} \mathrm{O}\right)$ & $\mathbf{0}$ & $\mathbf{1}$ & $\mathbf{5}$ & $\mathbf{1 0}$ & $\mathbf{5 0}$ & $\mathbf{1 0 0}$ \\
Carbono $\left(\mathrm{g} \mathrm{kg}^{-1}\right)$ & $8,3 \pm 0,1 \mathrm{a}$ & $8,3 \pm 0,1 \mathrm{a}$ & $8,3 \pm 0,1 \mathrm{a}$ & $8,3 \pm 0,1 \mathrm{a}$ & $8,3 \pm 0,1 \mathrm{a}$ & $8,4 \pm 0,1 \mathrm{a}$ \\
Nitrogênio $\left(\mathrm{g} \mathrm{kg}^{-1}\right)$ & $453 \pm 20 \mathrm{a}$ & $467 \pm 8,0 \mathrm{a}$ & $468 \pm 16,0 \mathrm{a}$ & $441 \pm 4,0 \mathrm{a}$ & $469 \pm 14,0 \mathrm{a}$ & $469 \pm 27,0 \mathrm{a}$ \\
Fósforo $\left(\mathrm{g} \mathrm{kg}^{-1}\right)$ & $12,8 \pm 0,4 \mathrm{a}$ & $13,3 \pm 0,4 \mathrm{a}$ & $13,8 \pm 0,5 \mathrm{a}$ & $13,1 \pm 0,4 \mathrm{a}$ & $13,6 \pm 0,4 \mathrm{a}$ & $14,1 \pm 0,8 \mathrm{a}$ \\
Potássio $\left(\mathrm{g} \mathrm{kg}^{-1}\right)$ & $4,2 \pm 0,1 \mathrm{a}$ & $4,2 \pm 0,2 \mathrm{a}$ & $4,4 \pm 0,1 \mathrm{a}$ & $4,3 \pm 0,1 \mathrm{a}$ & $4,4 \pm 0,2 \mathrm{a}$ & $4,3 \pm 0,1 \mathrm{a}$ \\
Cálcio $\left(\mathrm{g} \mathrm{kg}^{-1}\right)$ & $7,3 \pm 0,4 \mathrm{a}$ & $7,1 \pm 0,1 \mathrm{a}$ & $7,7 \pm 0,1 \mathrm{a}$ & $7,4 \pm 0,3 \mathrm{a}$ & $7,4 \pm 0,4 \mathrm{a}$ & $7,6 \pm 0,3 \mathrm{a}$ \\
Magnésio $\left(\mathrm{g} \mathrm{kg}^{-1}\right)$ & $13,8 \pm 0,3 \mathrm{a}$ & $14,7 \pm 0,8 \mathrm{ab}$ & $15,1 \pm 0,4 \mathrm{bc}$ & $14,4 \pm 0,6 \mathrm{ab}$ & $15,8 \pm 0,5 \mathrm{bc}$ & $15,8 \pm 0,6 \mathrm{c}$ \\
Relação C/N & $4,9 \pm 0,1 \mathrm{a}$ & $4,9 \pm 0,2 \mathrm{a}$ & $5,0 \pm 0,1 \mathrm{a}$ & $5,1 \pm 0,1 \mathrm{a}$ & $5,2 \pm 0,2 \mathrm{ab}$ & $5,4 \pm 0,1 \mathrm{~b}$ \\
\hline
\end{tabular}

Valores seguidos de mesma letra não diferem significativamente entre si, nas linhas, pelo teste de Duncan $(\mathrm{P}<0,05)$

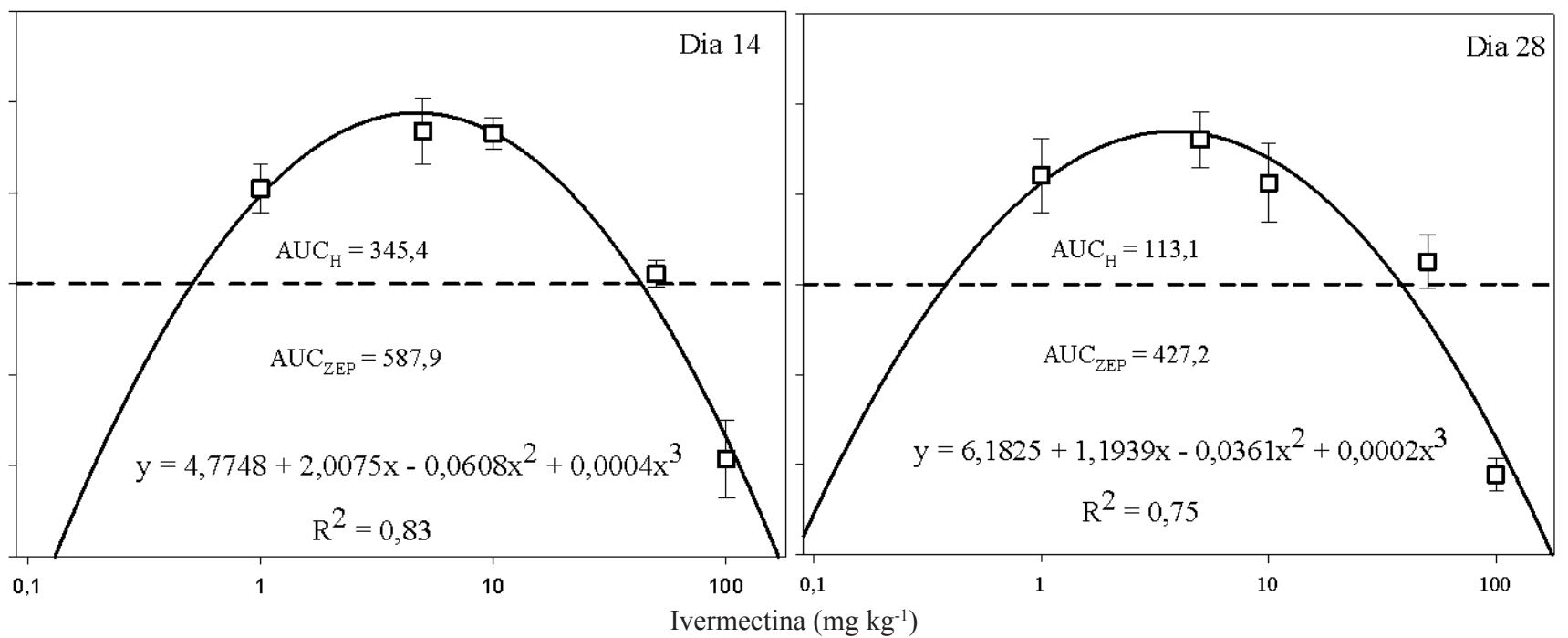

Figura 2. Efeito hormético sobre a atividade relativa da enzima fosfatase alcalina (Falc) em Eisenia foetida aos 14 e 28 dias de exposição a diferentes concentrações de ivermectina. Os dados são demonstrados como média \pm desvio padrão. $A \cup C_{H}$ representa a área sob a curva hormética e $A \cup C_{Z E P}$ indica a área sob a curva do ponto de equivalência zero 
desta classe estão envolvidas na diferenciação, na proliferação, na sinalização celular, na meiose, na mitose e na regulação da síntese do DNA, em diversos tipos de organimo (Fernandes et al., 2008).

\section{Conclusões}

1. A ivermectina se apresenta tóxica em altas concentrações no esterco bovino utilizado como substrato teste, mas não mostrou efeitos adversos sobre o crescimento, a reprodução nem quanto à sobrevivência de minhocas em baixos níveis.

2. Não há alterações significativas sobre a atividade microbiana nem na maioria dos parâmetros químicos do vermicomposto entre os grupos tratados com ivermectina e o grupo controle.

3. Observa-se efeito hormético induzido pela ivermectina sobre a taxa de desenvolvimento e atividade relativa da enzima fosfatase alcalina de Eisenia foetida.

\section{Agradecimentos}

Este estudo foi financiado por PROPPEX/URCAMP e PPGSPAF/UFPel.

\section{Literatura Citada}

Aira, M.; Domínguez, J. Microbial and nutrient stabilization of two animal manures after the transit through the gut of the earthworm Eisenia fetida (Savigny, 1826). Journal of Hazardous Materials, v.161, p.1234-1238, 2009. http://dx.doi.org/10.1016/j.jhazmat.2008.04.073

Atiyeh, R. M.; Domínguez, J.; Subler, S.; Edwards, C. A. Changes in biochemical properties of cow manure during processing by earthworms (Eisenia andrei, Bouché) and the effects on seedling growth. Pedobiologia, v.44, p.709-724, 2000. http://dx.doi. org/10.1078/S0031-4056(04)70084-0

Blanckenhorn, W. F.; Puniamoorthy, N.; Schafer, M. A.; Scheffczyk, A.; Römbke J. Standardized laboratory tests with 21 species of temperate and tropical sepsid flies confirm their suitability as bioassays of pharmaceutical residues (ivermectin) in cattle dung. Ecotoxicology and Environmental Safety, v.89, p.21-28, 2013. http:// dx.doi.org/10.1016/j.ecoenv.2012.10.020

Calabrese, E. J. Paradigm lost, paradigm found: The re-emergence of hormesis as a fundamental dose response model in the toxicological sciences. Environmental Pollution, v.138, p.378-411, 2005. http:// dx.doi.org/10.1016/j.envpol.2004.10.001

Calabrese, E. J.; Blain, R. B. The hormesis database: The occurrence of hormetic dose responses in the toxicological literature. Regulatory Toxicology and Pharmacology, v.61, p.73-81, 2011. http://dx.doi. org/10.1016/j.yrtph.2011.06.003

Cedergreen, N. Is the growth stimulation by low doses of glyphosate sustained over time? Environmental Pollution, v.156, p.1099-1104, 2008. http://dx.doi.org/10.1016/j.envpol.2008.04.016

Deng, C.; Graham, R.; Shukla, R. Detecting and estimating hormesis using a model-based approach. Human and Ecological Risk Assessment, v.7, p.849-866, 2001. http://dx.doi.org/10.1080/20018091094691

Fernandes, J.; Amorim, R.; Azevedo, I.; Martins, M. J. In vitro modulation of alkaline phosphatase activity of Saccharomyces cerevisiae grown in low or high phosphate medium. Brazilian Journal of Medical and Biological Research, v.41, p.41-46, 2008. http://dx.doi.org/10.1590/S0100-879X2006005000198
Floate, K.; Wardhaugh, K.; Boxall, A.; Sherratt, T. N. Fecal residues of veterinary parasiticides: Nontarget effects in the pasture environment. Annual Review of Entomology, v.50, p.153-79, 2005. http://dx.doi.org/10.1146/annurev.ento.50.071803.130341

Gao, Y.; Sun, Z.; Liu, Y.; Sun, X.; Li, Y.; Bao, Y.; Wang, G. Effect of albendazole anthelmintics on the enzyme activities of different tissue regions in Eisenia fetida. European Journal of Soil Biology, v.43, p.246-251, 2007. http://dx.doi.org/10.1016/j.ejsobi.2007.08.046

Garg, V. K.; Yadav, Y. K.; Sheoran, A.; Chand, S.; Kaushik, P. Livestock excreta management through vermicomposting using an epigeic earthworm Eisenia foetida. Environmentalist, v.26, p.269-276, 2006. http://dx.doi.org/10.1007/s10669-006-8641-z

Gunn, A.; Sadd, J. W. The effect of ivermectin on the survival, behaviour and cocoon production of the earthworm Eisenia fetida. Pedobiologia, v.38, p.327-333, 1994.

Hackenberger, B. K.; Perkusic, J.; Stepi,c S. Effect of temephos on cholinesterase activity in the earthworm Eisenia fetida (Oligochaeta, Lumbricidae). Ecotoxicology and Environmental Safety, v.71, p.583589, 2008. http://dx.doi.org/10.1016/j.ecoenv.2007.11.008

Halley B.A.; Jacob T.A.; Lu A.Y. The environmental impact of the use of ivermectin. Environmental effects and fate. Chemosphere, v.18, p.1543-1563, 1989. http://dx.doi.org/10.1016/0045-6535(89)90045-3

Iglesias, L. E.; Fusé, L. A.; Lifschitz, A. L.; Rodríguez, E. M.; Sagüés, M. F.; Saumell, C. A. Environmental monitoring of ivermectin excreted in spring climatic conditions by treated cattle on dung fauna and degradation of faeces on pasture. Parasitology Research, v.108, p.1185-1191, 2011. http://dx.doi.org/10.1007/s00436-010-2161-y Iglesias, L. E.; Saumell, C. A.; Fernandez, A. S.; Fuse, L. A.; Lifschitz, A. L.; Rodriguez, E. M.; Steffan, P. E.; Fiel, C. A. Environmental impact of ivermectin excreted by cattle treated in autumn on dung fauna and degradation of faeces on pasture. Parasitology Research, v.100, p.93-102, 2006. http://dx.doi.org/10.1007/s00436-006-0240-x

Kaneda, S.; Yamashita, N.; Uchida, T.; Shimano, S.; Miyoshi, N.; Sasaki, M.; Enami, Y. Effects of ivermectin in dung pats on earthworm (Megascolecidae) populations and pat degradation in Japanese grassland. Applied Soil Ecology, v.31, p.280-285, 2006. http://dx.doi. org/10.1016/j.apsoil.2005.05.001

Krogh, K. A.; Jensen, G. G.; Schneider, M. K.; Fenner, K.; Halling-Sorensen, B. Analysis of the dissipation kinetics of ivermectin at different temperatures and in four different soils. Chemosphere, v.75, p.10971104, 2009. http://dx.doi.org/10.1016/j.chemosphere.2009.01.015

Lumaret, J. P.; Errouissi, F.; Floate, K.; Römbke, J.; Wardhaugh, K. A review on the toxicity and non-target effects of macrocyclic lactones in terrestrial and aquatic environments. Current Pharmaceutical Biotechnology, v.13, p.1004-1060, 2012. http:// dx.doi.org/10.2174/138920112800399257

Prabha, L. M.; Jayaraaj, I. A.; Jeyaraaj, R.; Srinivasa, R. Comparative studies on the digestive enzymes in the gut of earthworms, Eudrilus eugenia and Eisenia foetida. Indian Journal of Biotechnology, v.6, p.567-569, 2007.

Rombke, J.; Krogh, K. A.; Moser, T.; Scheffczyk, A.; Liebig, M. Effects of the veterinary pharmaceutical ivermectin on soil invertebrates in laboratory tests. Archives of Environmental Contamination and Toxicology, v.58, p.332-340, 2009. http://dx.doi.org/10.1007/ s00244-009-9414-8

Silva, C. D. da; Costa, L. M.; Matos, A. T.; Cecon, P. R.; Silva, D. D. Vermicompostagem de lodo de esgoto urbano e bagaço de cana-de-açúcar. Revista Brasileira de Engenharia Agrícola e Ambiental, v.6, p.487-491, 2002. http://dx.doi.org/10.1590/S141543662002000300018 
Sun, Y.; Diao, X.; Zhang, Q.; Shen, J. Bioaccumulation and elimination of avermectin B1a in the earthworms (Eisenia fetida). Chemosphere, v.60, p.699-704, 2005. http://dx.doi.org/10.1016/j. chemosphere.2005.01.044

Svendsen, T. S.; Hansen, P. E.; Sommer, C.; Martinussen, T.; Grønvold, J.; Holter, P. Life history characteristics of Lumbricus terrestris and effects of the veterinary antiparasitic compounds ivermectin and fenbendazole. Soil Biology \& Biochemistry, v.37, p.927-936, 2005. http://dx.doi.org/10.1016/j.soilbio.2004.10.014

Tedesco, M. J.; Gianello, C.; Bissani, C. A.; Bohnen, H.; Volkweiss, S. J. Análises de solo, plantas e outros materiais. 2.ed. Porto Alegre: Universidade Federal do Rio Grande do Sul, 1995. 174p.

Ueda, M.; Asano, T.; Nakazawa, M.; Miyatake, K.; Inouye, K. Purification and characterization of novel raw-starch-digesting and cold-adapted $\alpha$-amylases from Eisenia foetida. Comparative Biochemistry and Physiology, Part B, v.150, p.125-130, 2008.
Wang, Y.; Cang, T.; Zhao, X.; Yu, R.; Chen, L.; Wu, C.; Wang, Q. Comparative acute toxicity of twenty-four insecticides to earthworm, Eisenia fetida. Ecotoxicology and Environmental Safety, v.79, p.122128, 2012. http://dx.doi.org/10.1016/j.ecoenv.2011.12.016

Yadav, A.; Garg, V. K. Recycling of organic wastes by employing Eisenia fetida. Bioresource Technology, v.102, p.2874-2880, 2011. http:// dx.doi.org/10.1016/j.biortech.2010.10.083

Zhang, Y.; Shen, G.; Yu, Y.; Zhu, H. The hormetic effect of cadmium on the activity of antioxidant enzymes in the earthworm Eisenia fetida. Environmental Pollution, v.157, p.3064-3068, 2009. http:// dx.doi.org/10.1016/j.envpol.2009.05.039

Zhu, X. W.; Liu, S. S.; Qin, L. T.; Chen, F.; Liu, H. L. Modeling nonmonotonic dose-response relationships: Model evaluation and hormetic quantities exploration. Ecotoxicology and Environmental Safety, v.89, p.130-136, 2013. http://dx.doi.org/10.1016/j. ecoenv.2012.11.022 